\title{
Modular forms vanishing on the hyperelliptic locus
}

\author{
By Riccardo Salvati MANNI \\ (Received November 14, 2001) \\ (Revised November 12, 2002) \\ (from Nagoya Mathematical Journal)
}

\section{Introduction}

Let $\mathbb{H}_{g}$ be the Siegel upper half space, i.e. the set of complex symmetric matrices $Z$ whose imaginary part is positive definite and $S p(2 g, \mathbb{R})$ be the real symplectic group. $S p(2 g, \mathbb{R})$ acts transitively on $\mathbb{H}_{g}$ via

$$
\sigma \cdot Z=(A Z+B)(C Z+D)^{-1}
$$

where $\sigma=\left(\begin{array}{ll}A & B \\ C & D\end{array}\right) \in S p(2 g, \mathbb{R})$.

Let $\Gamma_{g}$ denote the Siegel modular group $S p(2 g, \mathbb{Z})$ and for any positive integer $n$ let $\Gamma_{g}(n):=\left\{\sigma \equiv 1_{2 g} \bmod n\right\}$ denote the principal congruence subgroup of $\Gamma_{g}$, and $\Gamma_{g}(n, 2 n):=\left\{\sigma / \operatorname{diag}\left(A^{t} B\right) \equiv \operatorname{diag}\left(C^{t} D\right) \equiv 0 \bmod 2 n\right\}$ the Igusa group; then we shall denote by $\mathcal{A}_{g}, \mathcal{A}_{g}(n), \mathcal{A}_{g}(n, 2 n)$ respectively, the quotient space of $\mathbb{H}_{g}$ by these groups. They are the moduli space of principally polarized abelian varieties with or without a level $n$ (theta) structure. They have dimension equal to $(1 / 2) g(g+1)$. We can consider the moduli space $\mathcal{H}_{g}$ of hyperelliptic curves of genus $g$ embedded in $\mathcal{A}_{g}$ and we put $\mathcal{H}_{g}(n)$ and $\mathcal{H}_{g}(n, 2 n)$ equal to the preimage of the projection map. The dimension of these spaces is $2 g-1$.

The problem of giving a characterization for all genera of the hyperelliptic locus in term of vanishing of Thetanullwerte goes back to Thomae, cf. [Th]. He showed that if $Z$ is a hyperelliptic point then a certain number of thetanullwerte, whose characteristic satisfy certain rules, vanish. Only in 1984, Mumford, using the method of dynamical systems, proved that the vanishing of these thetanullwerte and the non vanishing of the remaining characterizes the hyperelliptic locus, cf. [M]. Then in $[\mathrm{P}]$, Poor, using classical methods, namely crossratio, reproved Mumford's result, giving also as light improvement regarding the boundary of this locus. We have to say that the vanishing locus of these thetanullwerte is defined in $\mathcal{A}_{g}(2)$.

Meanwhile several authors [D-O], [Ts], [S], independently, counted the irreducible components of $\mathcal{H}_{g}(2)$, proved that this number does not change, when one 
replaces the level 2 structure by a level $n$ structure, $n$ even, [Ts], whenever one changes the level, and showed that it is smooth [S]. At this point it make sense to ask if the characterization given in terms of vanishing of thetanullwerte is ideal theoretic or not. For technical reasons this question make sense on $\mathcal{A}_{g}(4,8)$, since all the thetanullwerte are modular forms relative to the same multiplier system only when the group is contained in $\Gamma_{g}(4,8)$.

This result has been obtained up to embedded components in [Ts].

In this paper we prove it completely, in a purely geometric way; in fact it will appear as a consequence of Riemann's singularity theorem, more precisely, we can say, that it is implicitely contained in the milestone paper [A-M].

Since we shall show that this space is locally complete intersection, we shall exhibit a set of thetanullwerte candidate to define the components of the hyperelliptic locus as complete intersections, up to some natural components coming from the abelian varieties with reducible theta divisor.

\section{The irreducible components}

In this section we first recall some basic facts about characteristics and the action of the symplectic group.

A characteristic $m$ is a column vector in $\mathbb{Z}^{2 g}$, with $m^{\prime}$ and $m^{\prime \prime}$ as first and second entry vectors. We put

$$
e(m)=(-1)^{t} m^{\prime} m^{\prime \prime}
$$

and we say that $m$ is even or odd according as $e(m)=1$ or -1 .

For any triplet $m_{1}, m_{2}, m_{3}$ of characteristics we set

$$
e\left(m_{1}, m_{2}, m_{3}\right)=e\left(m_{1}\right) e\left(m_{2}\right) e\left(m_{3}\right) e\left(m_{1}+m_{2}+m_{3}\right) .
$$

A sequence $m_{1}, \ldots, m_{r}$ of characteristics is essentially independent if for any choice of an even number of indices between 1 and $r$ we have that the sum of the corresponding characteristics is not congruent to zero mod 2 .

The unique action of $\Gamma_{g}$ on the set of characteristics mod 2 keeping invariant (2), (3) and the condition of being essentially independent is defined by

$$
\sigma \cdot m:=\left(\begin{array}{cc}
D & -C \\
-B & A
\end{array}\right)\left(\begin{array}{c}
m^{\prime} \\
m^{\prime \prime}
\end{array}\right)+\left(\begin{array}{c}
\operatorname{diag}\left(C^{t} D\right) \\
\operatorname{diag}\left(A^{t} B\right)
\end{array}\right)
$$

In the following we shall often consider characteristics with 0 and 1 as entries. In this situation a special role is played by sequences of characteristics that form a fundamental system, i.e. a sequence of $2 g+2$ characteristics satisfying the condition of being azygetic, i.e.

$$
e\left(m_{i}, m_{j}, m_{k}\right)=-1
$$


for all indeces $1 \leq i<j<k \leq 2 g+2$.

The fact that fundamental systems exist and are all conjugate under an extension of $\Gamma_{g}$, the Theta Group, is classical. Among the fundamental systems we have the special fundamental systems that are characterized by the property of having $g$ odd characteristics and $g+2$ even. The special fundamental systems are all conjugate under the action of $\Gamma_{g}$, cf. [F].

Let $X=V / \Lambda$ be an abelian variety of dimension $g, L$ a symmetric line bundle inducing a principal polarization $H$ on $X$, i.e. $\operatorname{dim} H^{0}(X, L)=1$. Assume that the period matrix of $X$ is $\left(Z, 1_{g}\right), Z \in \mathbb{H}_{g}$.

We shall denote by $X[2]$ the 2 torsion points of $X$. For $x=Z m^{\prime} / 2+m^{\prime \prime} / 2$ in $X[2]$, i.e. $m^{\prime}, m^{\prime \prime} \in \mathbb{Z}^{g}$, we shall denote by $t_{x}$ the translation associated with $x$. We remark that $t_{x}^{*} L$ is still a symmetric line bundle and we have that the theta function of characteristic $m=\left(m^{\prime}, m^{\prime \prime}\right)$ and modulus $Z$

$$
\vartheta_{m}(Z, z)=\sum_{p \in \mathbb{Z}^{g}} \underline{e}\left((1 / 2)^{t}\left(p+m^{\prime} / 2\right) Z\left(p+m^{\prime} / 2\right)+{ }^{t}\left(p+m^{\prime} / 2\right)\left(z+m^{\prime \prime} / 2\right)\right)
$$

is, up to a multiplicative constant, the unique section of the above line bundle. Here $\underline{e}(\quad)$ stands for $\exp (2 \pi i)$. From these facts it follows that theta functions depend on the characteristic mod 2 and there is the following relation between them

$$
\begin{aligned}
& \vartheta_{n+m}(Z, z) \\
& =\underline{e}\left((1 / 2)^{t}\left(m^{\prime} / 2\right) Z\left(m^{\prime} / 2\right)+{ }^{t}\left(m^{\prime} / 2\right)\left(z+m^{\prime \prime} / 2\right)\right) \underline{e}\left((1 / 2)^{t} m^{\prime} n^{\prime \prime}\right) \vartheta_{n}(Z, z+x) .
\end{aligned}
$$

We set $\Theta_{x}$ equal to the divisor of $\vartheta_{m}(Z, z)$; in particular we write $\Theta$ for the divisor of $\vartheta_{0}(Z, z)$.

The theta function is even or odd according as $e(m)$ is equal to 1 or -1 . We set $\theta_{m}:=\vartheta_{m}(Z, 0)$, this, as function on $\mathbb{H}_{g}$, is not identically zero if and only if the characteristic is even and it is called Thetanullwert.

We set $y=Z n^{\prime} / 2+n^{\prime \prime} / 2$, then at all points

$$
x \in \Theta_{y} \cap X[2]
$$

the following properties hold:

$$
\begin{aligned}
& \frac{\partial \vartheta_{n}}{\partial Z_{i j}}(Z, x) \\
& \left.\quad=\underline{e}\left(-(1 / 2)\left({ }^{t}\left(m^{\prime} / 2\right) Z\left(m^{\prime} / 2\right)+{ }^{t} m^{\prime} n^{\prime \prime}\right)\right)-{ }^{t}\left(m^{\prime} / 2\right) m^{\prime \prime} / 2\right) \frac{\partial \vartheta_{n+m}}{\partial Z_{i j}}(Z, 0)
\end{aligned}
$$

for all $i \leq j \leq g$.

We remark that in this formula we used the fact that

$$
\vartheta_{n}(Z, x)=\vartheta_{n+m}(Z, 0)=0 .
$$


We recall that for any $\sigma \in \Gamma_{g}$ we have the following transformation formula

$$
\theta_{\sigma \cdot m}(\sigma \cdot Z)=k(\sigma) e(\phi(m, \sigma)) \operatorname{det}(C Z+D)^{\frac{1}{2}} \theta_{m}(Z)
$$

Here $k(\sigma)$ and $e(\phi(m, \sigma))$ are eighth root of the unity, the first depending only on $\sigma$ and the second also on the characteristic $m$.

As we said in the introduction we know that the vanishing of certain Thetanullwerte characterizes the hyperelliptic locus. We recall briefly this fact. Let $\mathcal{C}$ be an hyperelliptic curve, $Q_{0}, \ldots, Q_{2 g+1}$ be its ramification points, we denote by $x_{0}, \ldots, x_{2 g+1}$ their image under the Abel-Jacobi map in the abelian variety $X$, normalized in such way that $x_{0}=0$. We recall that these points are half periods, i.e. they belongs to $X[2]$; further we denote by $m_{0}, \ldots, m_{2 g+1}$, the characteristic associated to these points, namely $x_{i}=\left(\frac{1}{2}\right)\left(Z m^{\prime}+m^{\prime \prime}\right), i=1, \ldots, 2 g+1$.

The following facts are well known, cf. [I1]:

i)

$$
m_{0}, \ldots \ldots \ldots, m_{2 g+1}
$$

form a fundamental system and consequently any $2 g$ among the characteristics $m_{1}, \ldots \ldots \ldots, m_{2 g+1}$ are linearly independent $\bmod 2$;

ii) we set $b$ equal to the sum of odd $m_{i}$ among them and if we agree to express any characteristic $\bmod 2$ as $m_{i_{1}}+\cdots+m_{i_{k}}$ for $0 \leq k \leq g$ in which $1 \leq i_{1}<\cdots<i_{k} \leq 2 g+1$, then the characteristic $m+b$ is even or odd according as $k \equiv g, g+1 \bmod 4$ or $k \equiv g+2, g+3 \bmod 4$;

iii) when the characteristic $m+b$ is even we have

$$
\theta_{m+b}(Z) \neq 0 \quad \text { if and only if } k=g
$$

iv)

$$
\frac{\partial \theta_{m+b}}{\partial Z_{i j}}(Z) \neq 0
$$

for at least a pair of indices $(i, j)$ if and only if $k=g-3$ or $g-4$. This is equivalent to the fact that the corresponding half period is a double point of the divisor $\Theta$.

We remark that the conditions of non-vanishing of the Thetanullwerte in iii) and the vanishing of the remaining, with even characteristics, are given by the equations of an irreducible component of the hyperelliptic locus in the moduli space $\mathcal{A}_{g}(4,8)$, cf. [M].

We know that all components are conjugate from the action of the group $\Gamma_{g}$, therefore all results, that one obtains for a component, hold for the others, cf. [I1].

Now let us recall from [A-M], p. 201, some other basic facts about hyperelliptic curves. 
i) The canonical image of an hyperelliptic curve $C$ is a rational twisted curve $\Gamma$ in $\mathbb{P}^{g-1}$. Every complete linear series $g_{g-1}^{1}$ is of the form

$$
g_{2}^{1}+P_{1}+\cdots+P_{g-3}
$$

and conversely if $P_{1}, \ldots, P_{g-3}$ are generic this series is complete.

ii) The full system of quadrics through $\Gamma$ can be realized as follows; choosing $g-1$ linearly independent points $P_{1}, \ldots, P_{g-1}$ on the curve, then the $N=\frac{1}{2}(g-$ 1) $(g-2)$ quadrics of rank 3 , projecting $\Gamma$ from the space spanned by $g-3$ of the above points, are linearly independent and span the full system.

We remark that since $\Gamma$ is a rational twisted curve, any set of $g-1$ points $P_{1}, \ldots, P_{g-1}$ with $P_{i} \neq P_{j}$, if $i \neq j$, are linearly independent.

Now we can state the following result:

Theorem 1. Let $k$ be a positive integer, $1 \leq k \leq g, k \equiv g, g+1 \bmod 4$. Any irreducible component of $\mathcal{H}_{g}(4,8)$ is ideal theoretically defined by the vanishing of Thetanullwerte $\theta_{m+b}(Z)$ with $m=m_{i_{1}}+\cdots+m_{i_{k}}, k<g$ and the non-vanishing of the remaining.

Proof. It is enough to prove that along the points of an irreducible component we have $\frac{1}{2}(g-1)(g-2)$ characteristics $m_{l, t}, 1 \leq l<t \leq g-1$, with $m_{l, t}+b$ even, such that the rank of the matrix

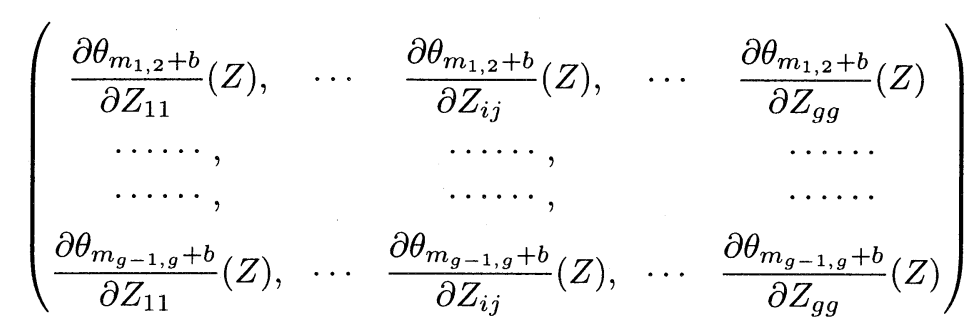

for $1 \leq i \leq j \leq g$ is equal to $\frac{1}{2}(g-1)(g-2)=\left(\frac{1}{2}\right) g(g+1)-2 g+1$.

We choose the points $P_{1}, \ldots, P_{g-1}$ on the rational twisted curve in the following way:

We take $P_{i}=Q_{i}$, for $i=1, \ldots, g-1$. From the remark above, or more specifically from Proposition 6.1 in [M], p. 95-96, it follows that the linear series

$$
g_{2}^{1}+Q_{i_{1}}+\cdots+Q_{i_{g-3}}
$$

with $1 \leq i_{1}<i_{2}<\cdots<i_{g-3} \leq g-1$, are $g_{g-1}^{1}$ 's. Now we consider the described full system of quadrics related to any of these subsets of $g-3$ points. 
The remarkable fact is that the images of these points, under the Abel-Jacobi map, are points of order two on the abelian variety and the same is true for their (partial) sums.

Moreover the sum of any $(g-3)$ of them has multiplicity 2 in a suitable translate of $\Theta$, i.e. the divisor of $\vartheta_{b}(Z, z)$.

According to (5), we have

$$
\begin{aligned}
& \frac{\partial \vartheta_{m_{l}+m_{t}+m_{g}}}{\partial Z_{i j}}(Z, 0) \\
& =c \frac{\partial \vartheta_{b}}{\partial Z_{i j}}\left(Z, x_{1}+\cdots+\hat{x}_{l}+\cdots+\hat{x}_{t}+\cdots+x_{g-1}\right)
\end{aligned}
$$

with $c$ a non zero constant and the convention that $\hat{x}_{l}$ means that we omit this term from the sum.

These are the coefficients of the related quadric, cf. [A-M] p. 210-212.

But these are also the entries of the rows of the matrix (6), thus the rank of the matrix is equal to the dimension of full system of quadrics through $\Gamma$.

We remark that we reproved the smoothness of the hyperelliptic locus in $\mathcal{A}_{g}(4,8)$. Obviously the smoothness implies that the hyperelliptic locus is locally complete intersection, here in the proof is also given explicitly a set of $\frac{1}{2}(g-1)(g-2)$ Thetanullwerte expressing each irreducible component as local complete intersection in $\mathcal{A}_{g}(4,8)$.

Let us conclude with some remarks. First we can consider also the closure of this locus in $\mathcal{A}_{g}(4,8)$, i.e. we do not require the non vanishing of the remaining Thetanullwerte, then also in this case it has been proved in [Ts] that the closure is smooth.

Moreover one could ask if these components are complete intersections. In genus 3 case this is trivial, since we have only a vanishing Thetanullwert, but already in genus 4 this fact becomes not trivial, in fact Igusa, cf. [I2], proved that the vanishing of 3 even Thetanullwerte whose characteristics form an azygetic triplet implies that the point in $\mathcal{A}_{g}(4,8)$ is hyperelliptic or reducible, i.e. there exists $\sigma \in \Gamma_{g}$ such that

$$
\sigma \cdot Z=\left(\begin{array}{cc}
Z_{1} & 0 \\
0 & Z_{2}
\end{array}\right), \quad Z_{i} \in \mathbb{H}_{g_{i}}, g_{1}+g_{2}=g
$$

For higher genera, with the best of our knowledge, the only fact that is known is that we always have some ugly component coming from the reducible points, this will be illustrated from the next example. 
We give explicitly a fundamental system of characteristics, so it will be easy to write down local equations for a component of $\mathcal{H}_{g}(4,8)$. We set

$$
\left(m_{0}, \ldots, m_{2 g+1}\right)=\left(\begin{array}{cccc}
0 & 1_{g} & 1_{g} & 0 \\
0 & \delta_{g} & 0 & \delta_{g}
\end{array}\right) .
$$

Here $\delta_{g}$ denotes the $g \times g$ matrix whose entries are $\delta_{i j}=1$ for $1 \leq i \leq j \leq g$, $\delta_{i j}=0$ for $i>j$ and 0 is a column vector. (Caution: here we have made an abuse of notations, since the entries in the same column do not have the same size, we recall that the columns are $2 g+2$ ).

Thus according to the choices that we made before we get the following even characteristics

$$
m_{i j}=m_{g}+m_{i}+m_{j} \quad 0<i<j<g ;
$$

whose associated Thetanullwerte $\theta_{m_{i j}}(Z)$ give local equations for an irreducible component of $\mathcal{H}_{g}(4,8)$.

We observe that they vanish on points

$$
Z=\left(\begin{array}{cc}
\tau & 0 \\
0 & w
\end{array}\right), \quad \tau \in \mathbb{H}_{g-1}, \quad \tau \in \mathbb{H}_{1},
$$

in fact, setting $n_{i j}$ equal to the characteristic obtained from $m_{i j}$ deleting the $g$-th and the $2 g$-th entry that are equal to 1 , we get

$$
\theta_{m_{i j}}\left(\begin{array}{cc}
\tau & 0 \\
0 & w
\end{array}\right)=\theta_{n_{i j}}(\tau) \theta_{11}(w)=0
$$

\section{References}

[A-M] A. Andreotti and A. Mayer, On period relations for abelian integrals on algebraic curves, Ann. Scuola Norm. Sup. Pisa, 21 (1967), 189-238.

[D-O] I. Dolgachev and D. Ortland, Point sets in projective spaces and theta functions, Asterisque, 165 (1988).

[ F ] J. Fay, On the Riemann-Jacobi formula, Nachr. Akad. Wiss. Göttingen, 5 (1979), 61-73.

[I1] J. Igusa, Modular forms and projective invariants, Amer. J. Math., 89 (1967), 817-855.

[I2] J. Igusa, On the irreducibility of Schottky's divisor, J. Fac. Sci. Univ. Tokyo, 28 (1982), 531-545.

[M] D. Mumford, Tata Lectures on Theta 2, Progr. Math., 43, Birkhäuser, Boston-BaselStuttgart (1984).

[P] C. Poor, The hyperelliptic locus, Duke Math. J., 76 (1994), 809-884.

[S] R. Sasaki, A remark on the moduli space of hyperelliptic period matrices with level 2 structure, Arch. Math., 62 (1994), 83-87.

[Th] J. Thomae, Beitrag zur Bestimmung von $\vartheta(0,0, \ldots, 0)$ durch die Klassenmoduln algebraischer Funktionen, J. Reine Angew. Math., 71 (1870), 201-222. 
[Ts] S. Tsuyumine, Thetanullwerte on the moduli space of curves and hyperelliptic loci, Math. Z., 207 (1991), 539-568.

\author{
Dipartimento di Matematica \\ UnIVERsità di Roma \\ Piazzale Aldo Moro, 2 \\ I-00185 Roma, ItTaLy \\ E-mail: salvati@mat.uniroma1.it
}

